\title{
Symmetric Chain Decompositions and the Strong Sperner Property for Noncrossing Partition Lattices
}

\author{
Henri Mühlel] \\ LIX, École Polytechnique, F-91128 Palaiseau, France
}

\begin{abstract}
We prove that the noncrossing partition lattices associated with the complex reflection groups $G(d, d, n)$ for $d, n \geq 2$ admit a decomposition into saturated chains that are symmetric about the middle ranks. A consequence of this result is that these lattices have the strong Sperner property, which asserts that the cardinality of the union of the $k$ largest antichains does not exceed the sum of the $k$ largest ranks for all $k \leq n$. Subsequently, we use a computer to complete the proof that any noncrossing partition lattice associated with a well-generated complex reflection group is strongly Sperner, thus affirmatively answering a special case of a question of D. Armstrong. This was previously established only for the Coxeter groups of type $A$ and $B$.
\end{abstract}

Résumé. Nous prouvons que les treillis des partitions non-croisées associés aux groupes de réflexion complèxe $G(d, d, n)$, où $d, n \geq 2$, admettent une décomposition en chaînes saturées qui sont symétriques par rapport au rang moyen. Une conséquence de ce résultat est que ces treillis ont la propriété forte de Sperner qui affirme que le cardinal de l'union des $k$ antichaînes les plus grands ne dépasse pas la somme des $k$ rangs les plus grands pour tout $k \leq n$. Ensuite, nous utilisons un ordinateur pour compléter la preuve que chaque treillis des partitions non-croisées associé à un groupe de réflexion complèxe bien engendré a la propriété forte de Sperner. Ce résultat résout un cas spécial d'un question de D. Armstrong, et avait été établi auparavant seulement pour les groupes de Coxeter de type $A$ et $B$.

Keywords. noncrossing partition lattices, well-generated complex reflection groups, symmetric chain decompositions, strongly Sperner posets

\section{Introduction}

A classical result due to E. Sperner asserts that the maximal size of a family of pairwise incomparable subsets of an $n$-element set (also called an antichain) is $\left(\begin{array}{c}n \\ \left\lfloor\frac{n}{2}\right\rfloor\end{array}\right)[28]$. This result was generalized by P. Erdôs, who showed that the maximal size of a $k$-family, i.e. a family of subsets of an $n$-element set that

\footnotetext{
${ }^{\dagger}$ Email: henri.muehledlix.polytechnique.fr. This work is supported by a Public Grant overseen by the French National Research Agency (ANR) as part of the "Investissements d'Avenir" Program (Reference: ANR-10-LABX-0098), and by Digiteo project PAAGT (Nr. 2015-3161D).
}

1365-8050 (C) 2016 Discrete Mathematics and Theoretical Computer Science (DMTCS), Nancy, France 
can be written as the union of at most $k$ antichains, is the sum of the largest $k$ binomial coefficients [16, Theorem 5]. The case $k=1$ clearly yields Sperner's original result.

We can easily rephrase these results in a poset-theoretical way, so that they can be read as "the maximal size of a union of $k$ antichains in the Boolean lattice does not exceed the sum of its $k$ largest rank numbers". If we now replace "the Boolean lattice" by "a graded poset", we arrive at a poset property that is usually called being $k$-Sperner. Moreover, posets that are $k$-Sperner for all $k$ not exceeding the length of the poset are called strongly Sperner. Examples of strongly Sperner posets are the Boolean lattices, the lattices of divisors of some integer [13], or the Bruhat posets associated with finite Coxeter groups [30]. Some posets that in general lack the Sperner property are geometric lattices [14], in particular lattices of set partitions of a sufficiently large set [9].

One method to prove that a graded poset is strongly Sperner is to check whether it can be decomposed into symmetric chains, i.e. saturated chains that are symmetric about the middle ranks. Despite the fact that the partition lattice is in general not Sperner, it has a well-behaved subposet, the lattice of noncrossing set partitions, which is indeed strongly Sperner. This property was shown by R. Simion and D. Ullmann by explicitly constructing a decomposition into symmetric chains in [27, Theorem 2]. In the late 1990s it was observed by several authors how the lattice of noncrossing set partitions can be viewed as a poset associated with the symmetric group [6, 7], and this construction was generalized algebraically to other reflection groups soon after so that nowadays there exists a "noncrossing partition lattice" for each wellgenerated complex reflection group $W$, denoted by $\mathcal{N C}_{W}$. According to the classification of irreducible complex reflection groups in [26], there is one infinite family whose members can be realized as groups of monomial $(n \times n)$-matrices whose non-zero entries are $(d e)^{\text {th }}$ roots of unity, and in which the product of the non-zero entries is a $d^{\text {th }}$ root of unity, and such groups are usually denoted by $G(d e, e, n)$. Moreover, there are 34 exceptional irreducible complex reflection groups, denoted by $G_{4}, G_{5}, \ldots, G_{37}$. The wellgenerated irreducible complex reflection groups fall into four categories: they are either isomorphic to $G(1,1, n)$ for some $n \geq 1$, to $G(d, 1, n)$ for some $d \geq 2, n \geq 1$, to $G(d, d, n)$ for some $d, n \geq 2$, or they are one of 26 exceptional groups. We remark that all irreducible finite Coxeter groups, namely reflection groups that can be realized over a real vector space, are well-generated. See [19, Example 2.11] for the details.

In the above notation, the groups $G(1,1, n)$ for $n \geq 1$ are precisely the symmetric groups, and the noncrossing partition lattice $\mathcal{N C}_{G(1,1, n)}$ is isomorphic to the classical lattice of noncrossing set partitions of an $n$-element set [6, 7]. Using a combinatorial construction predating the algebraic one, V. Reiner introduced noncrossing partition lattices for the hyperoctahedral groups (which are isomorphic to the groups $G(2,1, n), n \geq 1)$, and showed that these also have the strong Sperner property by giving a symmetric chain decomposition [24, Theorem 13]. D. Bessis showed in [4, 5] that the noncrossing partition lattice associated with a well-generated complex reflection group $W$ can be realized using the simple elements in the dual braid monoid of $W$, and it follows from this perspective that $\mathcal{N C}_{G(d, 1, n)} \cong \mathcal{N C}_{G(2,1, n)}$ for all $d \geq 2$ and $n \geq 1$. Thus when we restrict our attention to well-generated irreducible complex reflection groups, the question of whether or not all noncrossing partition lattices admit a symmetric chain decomposition is only open for the groups $G(d, d, n), d, n \geq 2$, and for the exceptional groups. In this article, we answer this question affirmatively for the groups $G(d, d, n)$ with $d, n \geq 2$.

Theorem 1 For any $d, n \geq 2$, the lattice $\mathcal{N C}_{G(d, d, n)}$ of $G(d, d, n)$-noncrossing partitions admits a symmetric chain decomposition. Consequently, it is strongly Sperner, rank-symmetric, and rank-unimodal.

The basic idea for our symmetric chain decomposition of $\mathcal{N C}_{G(d, d, n)}$ is that the elements in $G(d, d, n)$ 
can be viewed as certain permutations of $n$ integers in $d$ colors, and can therefore be grouped according to the image of the first integer in the first color. This is clearly a decomposition of the group (and consequently of the poset), and the subposets induced by the parts correspond to direct products of noncrossing partition lattices of type $G\left(d, d, n^{\prime}\right)$ or $G\left(1,1, n^{\prime}\right)$ for $n^{\prime}<n$. However, there exist parts in this decomposition that do not sit symmetrically in $\mathcal{N C}_{G(d, d, n)}$. We overcome this issue by slightly modifying this decomposition. We remark further that according to [3], E. Tzanaki obtained Theorem 1 for the case $d=2$ before. However, her proof has not appeared in print.

In his thesis [2], D. Armstrong generalized the noncrossing partition lattices associated with a Coxeter group $W$ by adding a parameter $m$, so that one obtains a certain partial order on the multichains of $\mathcal{N C}_{W}$ having length $m$, and this construction naturally extends to all well-generated complex reflection groups. Among many other things, he posed the question whether his $m$-divisible noncrossing partition posets, denoted by $\mathcal{N C}_{W}^{(m)}$, are strongly Sperner for any Coxeter group $W$ and any positive integer $m$ [2, Open Problem 3.5.12]. Our Theorem 1 together with a computer verification in the exceptional types answers this question affirmatively for $m=1$. A key tool for making the computer verification feasible is Proposition 15, which relates the strong Sperner property of a given graded poset to the Sperner property of certain rank-selected subposets.

Theorem 2 For any well-generated complex reflection group, the lattice $\mathcal{N C}_{W}$ is strongly Sperner, ranksymmetric, and rank-unimodal.

The poset $\mathcal{N C}_{W}^{(m)}$ can be viewed as an order ideal in the $m$-fold direct product of $\mathcal{N C}_{W}$ with itself. Since $\mathcal{N C}_{W}$ is rank-unimodal and rank-symmetric, the strong Sperner property is in this case preserved under taking direct products, and it follows that $\mathcal{N C}_{W}^{(m)}$ is an order ideal in a strongly Sperner poset. Can this connection be used to conclude the strong Sperner property for $\mathcal{N C}_{W}^{(m)}$ ? Since for $m>1$ the poset $\mathcal{N C}_{W}^{(m)}$ is not rank-symmetric anymore, we cannot expect the existence of a symmetric chain decomposition.

This document constitutes an extended abstract of [21], and we have therefore omitted many proofs and computations. (The omitted details can of course be found in the full version.) The layout of this paper is as follows. In Section 2, we recall the necessary notions, in particular the definitions of strongly Sperner posets (Section 2.1), complex reflection groups (Section 2.2), and noncrossing partitions (Section 2.4). In Section 3 we prove Theorem 1 . To accomplish that, we first investigate a straightforward generalization of the decomposition of $\mathcal{N C}_{G(1,1, n)}$ from [27, Theorem 2] to $\mathcal{N C}_{G(d, d, n)}$ for a certain choice of Coxeter element (Section 3.2). Subsequently, in Section 3.3. we modify this decomposition in order to make it a suitable starting point for a symmetric chain decomposition of $\mathcal{N C}_{G(d, d, n)}$. In Section 4 we conclude this extended abstract with the proof of Theorem 2

\section{Preliminaries}

In this section we recall the necessary definitions that we use in this article. For further background on partially ordered sets we recommend [12]; an excellent introduction to the Sperner property and related subjects is [1]. An extensive textbook on complex reflection groups is [19], and a recent exposition on Coxeter elements is [25]. Throughout the paper we use the abbreviation $[n]=\{1,2, \ldots, n\}$ for an integer $n$, and we consider only finite posets. 


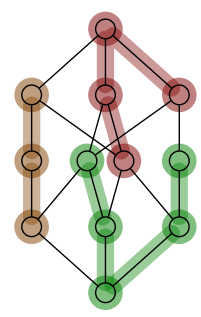

(a) A decomposition of a graded poset into three parts.

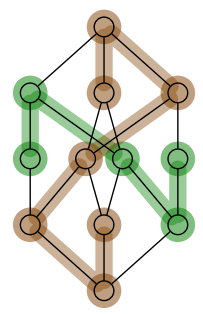

(b) A symmetric decomposition of the poset in Figure 1(a)

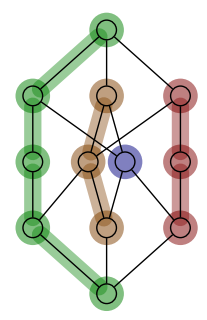

(c) A symmetric chain decomposition of the poset in Figure 1(a) into four chains, one of which is a singleton sitting on the middle rank.

Fig. 1: Examples of poset decompositions.

\subsection{Partially Ordered Sets}

Let $\mathcal{P}=(P, \leq)$ be a partially ordered set (poset for short). Given two elements $p, q \in P$, we say that $q$ covers $p$ if $p<q$ and there exists no $x \in P$ with $p<x<q$. In this case, we also say that $p$ is covered by $q$ or that $p$ and $q$ form a covering, and we usually write $p \lessdot q$. If $\mathcal{P}$ has a least element, say $\hat{0}$, then any element $p \in P$ with $\hat{0} \lessdot p$ is an atom of $\mathcal{P}$. Dually, if $\mathcal{P}$ has a greatest element, say $\hat{1}$, then any element $p \in P$ with $p \lessdot \hat{1}$ is a coatom of $\mathcal{P}$. An interval of $\mathcal{P}$ is a subset of $P$ that can be written in the form $[p, q]=\{x \in P \mid p \leq x \leq q\}$ for some $p, q \in P$ with $p \leq q$. A chain of $\mathcal{P}$ is a subset of $P$ that can be written as $C=\left\{p_{1}, p_{2}, \ldots, p_{s}\right\}$ such that $p_{1}<p_{2}<\cdots<p_{s}$, and the length of a chain is its cardinality minus one. A chain is saturated if it is a sequence of coverings. A saturated chain is maximal if it contains a minimal and a maximal element of $\mathcal{P}$. The length of $\mathcal{P}$ is the maximal length of a maximal chain, and we usually denote this by $\ell(\mathcal{P})$. A poset is graded if all maximal chains have the same length, and we can then assign a rank to each element $x \in P$, namely the length of a maximal chain from a minimal element in $\mathcal{P}$ to $x$. We usually write $\operatorname{rk}(x)$ for the rank of $x$. A lattice is a poset in which any two elements have a least upper bound and a greatest lower bound.

Given two posets $\mathcal{P}=\left(P, \leq_{P}\right)$ and $\mathcal{Q}=\left(Q, \leq_{Q}\right)$, the direct product is the poset $\mathcal{P} \times \mathcal{Q}=(P \times Q, \leq)$, where $\left(p_{1}, q_{1}\right) \leq\left(p_{2}, q_{2}\right)$ if and only if $p_{1} \leq_{P} p_{2}$ and $q_{1} \leq_{Q} q_{2}$. If we denote disjoint set union by $\uplus$, then, by abuse of notation, the disjoint union is the poset $\mathcal{P} \uplus \mathcal{Q}=(P \uplus Q, \leq)$, where $x \leq y$ if and only if either $x, y \in P$ and $x \leq_{P} y$ or $x, y \in Q$ and $x \leq_{Q} y$.

A decomposition of $\mathcal{P}$ is a partition of the ground set of $\mathcal{P}$ into pairwise disjoint subsets, namely $P=D_{1} \uplus D_{2} \uplus \cdots \uplus D_{s}$. A decomposition of a graded poset is symmetric if for each $i \in[s]$ the part $D_{i}$ induces a connected ${ }^{(i)}$ subposet with the property that for each minimal element $p$ of $D_{i}$ there exists a unique maximal element $q$ of $D_{i}$ with $\operatorname{rk}(p)+\operatorname{rk}(q)=\ell(\mathcal{P})$. A saturated chain $C=\left\{p_{1}, p_{2}, \ldots, p_{s}\right\}$ is symmetric if $\operatorname{rk}\left(p_{1}\right)+\operatorname{rk}\left(p_{s}\right)=\ell(\mathcal{P})$, and a symmetric chain decomposition of $\mathcal{P}$ is a decomposition of $P$ into symmetric chains. See Figure 1 for some examples.

An antichain of $\mathcal{P}$ is a subset of $P$ that consists of pairwise incomparable elements. Now suppose that

(i) Here "connected" means that the Hasse diagram of $\left(D_{i}, \leq\right)$ is an induced subgraph of the Hasse diagram of $\mathcal{P}$, i.e. for all $x, y \in D_{i}$ we have $x \lessdot y$ in $\left(D_{i}, \leq\right)$ if and only if $x \lessdot y$ in $\mathcal{P}$. 


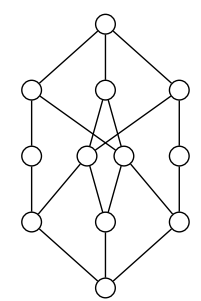

(a) A strongly Sperner poset.

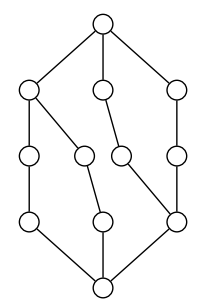

(b) A Sperner poset that is not 2 -Sperner.

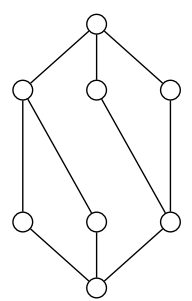

(c) A 2-Sperner poset that is not Sperner.

Fig. 2: Some illustrations of the Sperner property.

$\mathcal{P}$ is graded and has length $n$. The rank vector $\mathbf{N}_{\mathcal{P}}=\left(N_{0}, N_{1}, \ldots, N_{n}\right)$ of $\mathcal{P}$ is defined by $N_{i}=\mid\{p \in$ $P \mid \operatorname{rk}(p)=i\} \mid$ for $i \in\{0,1, \ldots, n\}$, and its entries are the rank numbers of $\mathcal{P}$. If $N_{i}=N_{n-i}$ for all $i$, then $\mathcal{P}$ is rank-symmetric, and if $N_{0} \leq N_{1} \leq \cdots \leq N_{j} \geq N_{j+1} \geq \cdots \geq N_{n}$ for some $j$, then $\mathcal{P}$ is rank-unimodal. A $k$-family is a subset of $P$ that can be written as a union of at most $k$ antichains of $\mathcal{P}$. A graded poset is $k$-Sperner if the size of a maximal $k$-family equals the sum of the $k$ largest rank numbers, and it is strongly Sperner if it is $k$-Sperner for all $k \in[n]$. See Figure 2 for some examples. The next result states the connection between symmetric chain decompositions and the strong Sperner property.

Theorem 3 ([15, Lemma 5.1.1 and Theorem 5.1.4]) If a graded poset admits a symmetric chain decomposition, then it is strongly Sperner, rank-symmetric, and rank-unimodal.

Graded posets that are strongly Sperner, rank-symmetric, and rank-unimodal are sometimes called Peck [31]. We further recall that the existence of a symmetric chain decomposition and the Peck property are both preserved under taking direct products.

Theorem 4 ([1, Theorem 3.6.1],[17]) If $\mathcal{P}$ and $\mathcal{Q}$ are two posets that admit a symmetric chain decomposition, then so does their direct product $\mathcal{P} \times \mathcal{Q}$.

Theorem 5 ([10, Theorem 1],[23, Theorem 3.2]) The direct product of two Peck posets is also Peck.

\subsection{Complex Reflection Groups}

A reflection is a unitary transformation $t$ on an $n$-dimensional complex vector space $V$ that has finite order and fixes a subspace of $V$ of codimension 1, the so-called reflecting hyperplane associated with $t$. A complex reflection group is a finite subgroup $W$ of the group of all unitary transformations on $V$ generated by reflections. If $W$ does not preserve a proper subspace of $V$, then $W$ is irreducible, and the rank of $W$ is the codimension of the space fixed by $W$. An irreducible complex reflection group is well-generated if it has rank $n$ and can be generated by $n$ reflections.

According to the classification of irreducible complex reflection groups due to G. C. Shephard and J. A. Todd [26], there exists one infinite family of such groups, parametrized by three integers $d, e, n$, whose members are usually denoted by $G(d e, e, n)$, as well as 34 exceptional groups, usually denoted by $G_{4}, G_{5}, \ldots, G_{37}$. The groups $G(d e, e, n)$ can be realized as groups of monomial $(n \times n)$-matrices, i.e. matrices with a unique non-zero entry in each row and in each column. For a monomial matrix to 
belong to $G(d e, e, n)$ its non-zero entries need to be $(d e)^{\text {th }}$ roots of unity, while the product of all its nonzero entries needs to be a $d^{\text {th }}$ root of unity. Consequently these groups possess a wreath product structure, see [19. Chapter 2.2] for the details. It follows from [22, Table 2] that there are three infinite families of well-generated irreducible complex reflection groups, namely $G(1,1, n)$ for $n \geq 1, G(d, 1, n)$ for $n \geq 1$ and $d \geq 2$, and $G(d, d, n)$ for $n, d \geq 2$, as well as 26 well-generated exceptional irreducible complex reflection groups.

\subsection{Coxeter Elements}

A vector $\mathbf{v} \in V$ is regular if it does not lie in any of the reflecting hyperplanes of $W$. If $\zeta$ is an eigenvalue of $w \in W$, and the corresponding eigenspace contains a regular vector, then we say that $w$ is $\zeta$-regular. The multiplicative order $d$ of $\zeta$ is a regular number for $W$. The $\zeta$-regular elements of $W$ form a single conjugacy class [29, Theorem 4.2].

A distinctive property of complex reflection groups is the fact that its algebra of invariant polynomials is again a polynomial algebra [11,26]. The degrees of a homogeneous choice of generators of this algebra yields a sequence of group invariants, the degrees of the group. If $W$ is a well-generated irreducible complex reflection group, then it follows from [18, Theorem $\mathrm{C}]$ that the largest degree is always a regular number for $W$, and we denote it by $h$. In that case we define a Coxeter element of $W$ to be any $\zeta$-regular element of order $h$ for some $h^{\text {th }}$ root of unity $\zeta$ [25, Definition 1.19].

\subsection{Noncrossing Partitions}

Let $W$ be a well-generated irreducible complex reflection group, and let $T \subseteq W$ denote the set of all reflections of $W$. The absolute length of $w \in W$ is defined by

$$
\ell_{T}(w)=\min \left\{k \mid w=t_{1} t_{2} \cdots t_{k} \text { for } t_{i} \in T\right\}
$$

and the absolute order on $W$ is the partial order $\leq_{T}$ defined by

$$
u \leq_{T} v \text { if and only if } \ell_{T}(v)=\ell_{T}(u)+\ell_{T}\left(u^{-1} v\right),
$$

for all $u, v \in W$. For any Coxeter element $\gamma \in W$, define the set of $W$-noncrossing partitions by

$$
N C_{W}(\gamma)=\left\{u \in W \mid \varepsilon \leq_{T} u \leq_{T} \gamma\right\}
$$

where $\varepsilon$ denotes the identity of $W$. The poset $\mathcal{N C _ { W }}(\gamma)=\left(N C_{W}(\gamma), \leq_{T}\right)$ is the lattice of $W$-noncrossing partitions, and its structure does not depend on the choice of $\gamma$ as the next result shows. We thus suppress the Coxeter element in the notation whenever it is not necessary.

Proposition 6 ([25, Corollary 1.6]) Let $W$ be a well-generated irreducible complex reflection group, and let $\gamma, \gamma^{\prime} \in W$ be two Coxeter elements. The corresponding posets $\mathcal{N C}_{W}(\gamma)$ and $\mathcal{N C}_{W}\left(\gamma^{\prime}\right)$ are isomorphic.

We refer the reader to [21, Section 2.4] for a historical account on the verification of the lattice property of $\mathcal{N C}_{W}$, as well as a list of other properties of this poset. 


\section{Decompositions of $\mathcal{N C}_{G(d, d, n)}$}

\subsection{The Setup}

In this section, we focus on the well-generated irreducible complex reflection group $G(d, d, n)$ for some fixed choice of $d, n \geq 2$. Recall from Section 2.2 that the elements of $G(d, d, n)$ can be realized as monomial $(n \times n)$-matrices whose non-zero entries are $d^{\text {th }}$ roots of unity and where the product of all non-zero entries is 1 . In this representation, we can view $G(d, d, n)$ as a subgroup of the symmetric group $\mathfrak{S}_{d n}$ acting on the set

$$
\left\{1^{(0)}, 2^{(0)}, \ldots, n^{(0)}, 1^{(1)}, 2^{(1)}, \ldots, n^{(1)}, \ldots, 1^{(d-1)}, 2^{(d-1)}, \ldots, n^{(d-1)}\right\}
$$

of $n$ integers with $d$ colors, where $k^{(s)}$ represents the column vector which has zeros in each entry except the $k^{\text {th }}$ one, and the $k^{\text {th }}$ entry is $\zeta^{s}$ for some primitive $d^{\text {th }}$ root of unity $\zeta$. Each $w \in G(d, d, n)$ satisfies

$$
w\left(k^{(s)}\right)=\pi(k)^{\left(s+t_{k}\right)} \quad \text { and } \quad \sum_{i=1}^{k} t_{k} \equiv 0 \quad(\bmod d),
$$

where $\pi \in \mathfrak{S}_{n}$ and the numbers $t_{k}$ depend only on $w$ and $k$. (Here, addition in the superscript is considered modulo $d$.) More precisely, the permutation $\pi$ is given by the permutation matrix that is derived from $w$ by replacing each non-zero entry by 1 , and the number $t_{k}$ is determined by the non-zero value in position $(k, \pi(k))$ of $w$. Consequently, we can decompose the elements of $G(d, d, n)$ into generalized cycles of the following form

$$
\left(\left(k_{1}^{\left(t_{1}\right)} \ldots k_{r}^{\left(t_{r}\right)}\right)\right)=\left(k_{1}^{\left(t_{1}\right)} \ldots k_{r}^{\left(t_{r}\right)}\right)\left(k_{1}^{\left(t_{1}+1\right)} \ldots k_{r}^{\left(t_{r}+1\right)}\right) \ldots\left(k_{1}^{\left(t_{1}+d-1\right)} \ldots k_{r}^{\left(t_{r}+d-1\right)}\right),
$$

and

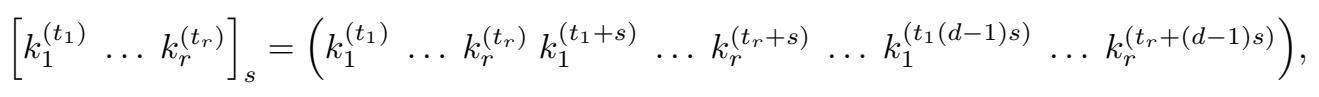

for $s \in[d-1]$. We call the first type a short generalized cycle and the second type a long generalized cycle, and we usually suppress the subscript 1 . In what follows we consider the Coxeter element $\gamma \in G(d, d, n)$ given by the product of the two long generalized cycles

$$
\gamma=\left[1^{(0)} 2^{(0)} \ldots(n-1)^{(0)}\right]\left[n^{(0)}\right]_{d-1} .
$$

See for instance [20, Section 3.3] for a justification that this is indeed a Coxeter element of $G(d, d, n)$. The next lemma characterizes the set $T_{\gamma}=T \cap N C_{W}(\gamma)$.

Lemma 7 ([20, Proposition 3.6]) Let $\gamma$ be the Coxeter element of $G(d, d, n)$ as defined in (4). Then we have

$$
T_{\gamma}=\left\{\left(\left(a^{(0)} b^{(s)}\right)\right) \mid 1 \leq a<b<n, s \in\{0, d-1\}\right\} \cup\left\{\left(\left(a^{(0)} n^{(s)}\right)\right) \mid 1 \leq a<n, 0 \leq s<d\right\} .
$$




\subsection{A First Decomposition}

It is the goal of this abstract to prove that the posets $\mathcal{N C}_{G(d, d, n)}(\gamma)$ admit a symmetric chain decomposition, and we accomplish this by generalizing the core idea of [27, Theorem 2]. There R. Simion and D. Ullmann prove that $\mathcal{N C}_{G(1,1, n)}(c)$ admits a symmetric chain decomposition where $c=\left(\begin{array}{llll}1 & 2 & \ldots & n\end{array}\right)$ is a long cycle in $G(1,1, n) \cong \mathfrak{S}_{n}$. For $i \in[n]$ define $R_{i}=\left\{u \in N C_{G(1,1, n)}(c) \mid u(1)=i\right\}$, and let $\mathcal{R}_{i}=\left(R_{i}, \leq_{T}\right)$. Moreover, let 2 denote the 2 -element chain. We have the following result.

Theorem 8 ([27, Theorem 2]) For $n>0$ we have $N C_{G(1,1, n)}(c)=\biguplus_{i=1}^{n} R_{i}$. Moreover, we have $\mathcal{R}_{1} \uplus$ $\mathcal{R}_{2} \cong \mathbf{2} \times \mathcal{N C}_{G(1,1, n-1)}$ and $\mathcal{R}_{i} \cong \mathcal{N C}_{G(1,1, i-2)} \times \mathcal{N C}_{G(1,1, n-i+1)}$ for $3 \leq i \leq n$. This decomposition is symmetric.

An immediate consequence of Theorem 8 is that $\mathcal{N C}_{G(1,1, n)}$ admits a symmetric chain decomposition. Recall from Section 3.1 that $G(d, d, n)$ can be realized as a subgroup of $\mathfrak{S}_{d n}$ permuting $n$ integers in $d$ colors. Therefore we define $R_{i}^{(s)}=\left\{u \in N C_{G(d, d, n)}(\gamma) \mid u\left(1^{(0)}\right)=i^{(s)}\right\}$ for $i \in[n]$ and $s \in$ $\{0,1, \ldots, d-1\}$. This is obviously a decomposition of $N C_{G(d, d, n)}(\gamma)$, but quite a lot of these parts are actually empty. The proof of the next statement can be found in [21]*Lemma 3.6.

Lemma 9 The sets $R_{1}^{(s)}$ and $R_{i}^{\left(s^{\prime}\right)}$ are empty for $2 \leq s<d$ as well as $2 \leq i<n$ and $1 \leq s^{\prime}<d-1$.

The next lemma describes the isomorphism type of the subposets of $\mathcal{N C}_{G(d, d, n)}(\gamma)$ induced by the nonempty sets $R_{i}^{(s)}$. Let us abbreviate $\mathcal{R}_{i}^{(s)}=\left(R_{i}^{(s)}, \leq_{T}\right)$. The proof of this statement is rather straightforward and thus omitted. See [21, Lemmas 3.7-3.12] for the details.

Lemma 10 The nonempty sets $R_{i}^{(s)}$ induce the following subposets of $\mathcal{N C}_{G(d, d, n)}(\gamma)$.

(i) The poset $\mathcal{R}_{1}^{(0)} \uplus \mathcal{R}_{2}^{(0)}$ is isomorphic to $2 \times \mathcal{N C}_{G(d, d, n-1)}$. Moreover, its least element has length 0 and its greatest element has length $n$.

(ii) The poset $\mathcal{R}_{n}^{(s)}$ is isomorphic to $\mathcal{N C}_{G(1,1, n-1)}$ for $0 \leq s<d$. Morever, its least element has length 1 and its greatest element has length $n-1$.

(iii) The poset $\mathcal{R}_{i}^{(0)}$ is isomorphic to $\mathcal{N C}_{G(d, d, n-i+1)} \times \mathcal{N C}_{G(1,1, i-2)}$ whenever $3 \leq i<n$. Moreover, its least element has length 1 and its greatest element has length $n-1$.

(iv) The poset $\mathcal{R}_{i}^{(d-1)}$ is isomorphic to $\mathcal{N C}_{G(1,1, n-i)} \times \mathcal{N C}_{G(d, d, i-1)}$ whenever $3 \leq i<n$. Moreover, its least element has length 1 and its greatest element has length $n-1$.

(v) The posets $\mathcal{R}_{1}^{(1)}$ and $\mathcal{R}_{2}^{(d-1)}$ are both isomorphic to $\mathcal{N C}_{G(1,1, n-2)}$. In the first case the least element has length 2 and the greatest element has length $n-1$, while in the second case the least element has length 1 and the greatest element has length $n-2$.

We observe that the induced subposet $\mathcal{R}_{1}^{(1)} \uplus \mathcal{R}_{2}^{(d-1)}$ of $\mathcal{N C}_{G(d, d, n)}(\gamma)$ is disconnected, see for instance Figure 3 (In this example $\mathcal{R}_{1}^{(1)}$ consists only of the coatom $\left[1^{(0)}\right]\left[3^{(0)}\right]_{4}$, and $\mathcal{R}_{2}^{(4)}$ consists only of the atom $\left(\left(1^{(0)} 2^{(4)}\right)\right)$, and these two elements are incomparable.) Furthermore, since both $R_{1}^{(1)}$ and $R_{2}^{(d-1)}$ do not sit in $\mathcal{N C}_{G(d, d, n)}(\gamma)$ symmetrically, this decomposition is not a suitable starting point to obtain a symmetric chain decomposition of $\mathcal{N C}_{G(d, d, n)}(\gamma)$. We overcome this issue in the next section. 


\subsection{A Second Decomposition}

It is straightforward to verify that $\mathcal{R}_{1}^{(1)}$ has least element $\left[1^{(0)}\right]\left[n^{(0)}\right]_{d-1}$ and greatest element

$$
\left[1^{(0)}\right]\left[n^{(0)}\right]_{d-1}\left(\left(2^{(0)} \ldots(n-1)^{(0)}\right)\right) \text {. }
$$

Therefore the map $x \mapsto\left(\left(1^{(0)} n^{(d-2)}\right)\right) x$ is an isomorphism from $\mathcal{R}_{1}^{(1)}$ to the interval

$$
E_{1}=\left[\left(\left(1^{(0)} n^{(d-1)}\right)\right),\left(\left(1^{(0)} n^{(d-1)}\right)\right)\left(\left(2^{(0)} \ldots(n-1)^{(0)}\right)\right)\right]_{T}
$$

in $\mathcal{N C}_{G(d, d, n)}(\gamma)$, which is isomorphic to $\mathcal{N C}_{G(1,1, n-2)}$ in its own right.

Analogously, we can show that $\mathcal{R}_{2}^{(d-1)}$ has least element $\left(\left(1^{(0)} 2^{(d-1)}\right)\right)$ and greatest element

$$
\left(\left(\left(1^{(0)} 2^{(d-1)} \ldots(n-1)^{(d-1)}\right)\right) .\right.
$$

Therefore the map $x \mapsto\left(\left(2^{(0)} n^{(0)}\right)\right) x$ is an isomorphism from $\mathcal{R}_{2}^{(d-1)}$ to the interval

$$
E_{2}=\left[\left(\left(1^{(0)} n^{(d-1)} 2^{(d-1)}\right)\right),\left(\left(1^{(0)} n^{(d-1)} 2^{(d-1)} \ldots(n-1)^{(d-1)}\right)\right)\right]_{T}
$$

in $\mathcal{N C}_{G(d, d, n)}(\gamma)$, which is again isomorphic to $\mathcal{N C}_{G(1,1, n-2)}$.

We observe that $E_{1}$ and $E_{2}$ are disjoint subsets of $R_{n}^{(d-1)}$, and therefore we may define $D_{1}=R_{1}^{(1)} \uplus$ $E_{1}, D_{2}=R_{2}^{(d-1)} \uplus E_{2}$, and $D=R_{n}^{(d-1)} \backslash\left(E_{1} \uplus E_{2}\right)$, as well as $\mathcal{D}_{1}=\left(D_{1}, \leq_{T}\right), \mathcal{D}_{2}=\left(D_{2}, \leq_{T}\right)$, and $\mathcal{D}=\left(D, \leq_{T}\right)$. The following lemma is now immediate.

Lemma 11 For $d, n \geq 2$, we have $D_{1} \uplus D_{2} \uplus D=R_{1}^{(1)} \uplus R_{2}^{(d-1)} \uplus R_{n}^{(d-1)}$. More precisely, $D_{1} \cong D_{2} \cong$ $2 \times \mathcal{N C}_{G(1,1, n-2)}$, and the least element in $D_{1}$ and $D_{2}$, respectively, has length 1 , while the greatest element in $D_{1}$ and $D_{2}$, respectively, has length $n-1$. Furthermore, $D \cong \biguplus_{i=3}^{n-1}\left(N C_{G(1,1, i-2)} \times N C_{G(1,1, n-i)}\right)$, and this decomposition is symmetric within $\mathcal{N C}_{G(d, d, n)}(\gamma)$.

We obtain the following result, which is illustrated in Figure 3

Theorem 12 For $d, n \geq 2$ the decomposition

$$
N C_{G(d, d, n)}(\gamma)=R_{1}^{(0)} \uplus R_{2}^{(0)} \uplus \biguplus_{i=3}^{n-1}\left(R_{i}^{(0)} \uplus R_{i}^{(d-1)}\right) \uplus \biguplus_{s=0}^{d-2} R_{n}^{(s)} \uplus D_{1} \uplus D_{2} \uplus D
$$

is symmetric. Consequently $\mathcal{N C}_{G(d, d, n)}(\gamma)$ admits a symmetric chain decomposition.

Proof: This follows from Lemmas 10 and 11 The claim on the existence of a symmetric chain decomposition now follows by induction on $n$ using Theorems 4 and 8 and Proposition 6 .

Proof of Theorem 1: Theorem 12 establishes the claim for the fixed Coxeter element $\gamma$ from (4), and in view of Proposition 6 , the claim holds for any Coxeter element of $G(d, d, n)$. 


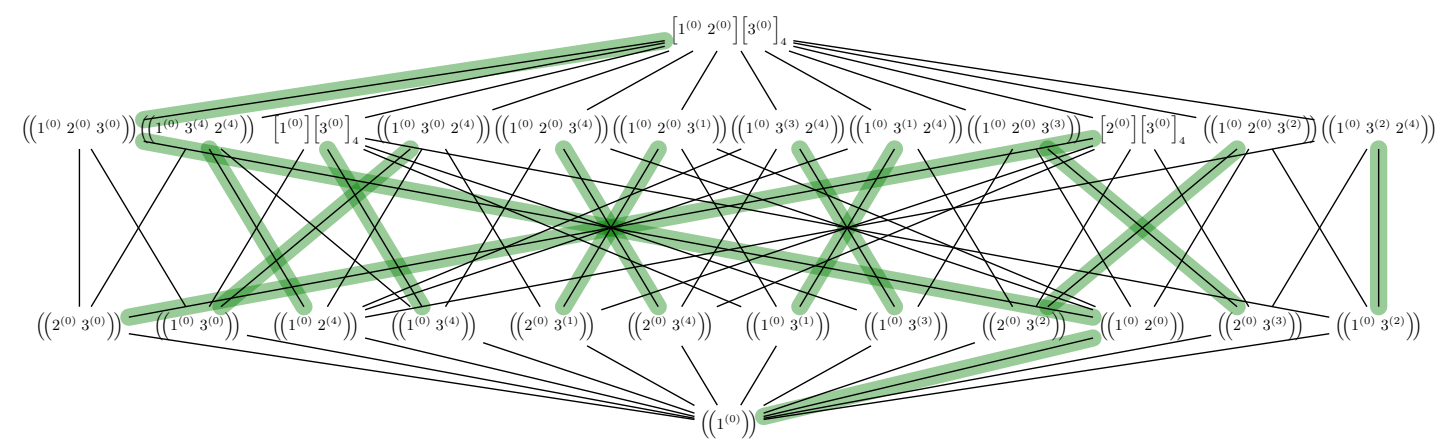

Fig. 3: The lattice $\mathcal{N C}_{G(5,5,3)}\left(\left[1^{(0)} 2^{(0)}\right]\left[3^{(0)}\right]_{4}\right)$. The symmetric chain decomposition coming from $[5$ is highlighted.

\section{The Remaining Cases}

Recall that there are three infinite families of well-generated irreducible complex reflection groups, namely $G(1,1, n)$ for $n \geq 1, G(d, 1, n)$ for $d \geq 2, n \geq 1$, and $G(d, d, n)$ for $d, n \geq 2$, as well as 26 exceptional well-generated complex reflection groups. Theorem 8 states that there exists a symmetric chain decomposition for $\mathcal{N C}_{G(1,1, n)}$, and we have proven in Theorem 1 that the same is true for $\mathcal{N C}_{G(d, d, n)}$. What about the other well-generated complex reflection groups? Recall a few more results.

Theorem 13 ([24, Theorem 13]) The lattice $\mathcal{N C}_{G(2,1, n)}$ admits a symmetric chain decomposition for any $n \geq 1$.

The next result follows from [5, Proposition 8.5] and [8, Table 1].

Proposition 14 For $d \geq 2, n \geq 1$, we have $\mathcal{N C}_{G(d, 1, n)} \cong \mathcal{N C}_{G(2,1, n)}$. Moreover, we have $\mathcal{N C}_{G_{25}} \cong$ $\mathcal{N C}_{G(1,1,4)}, \mathcal{N C}_{G_{26}} \cong \mathcal{N C}_{G(2,1,3)}$, and $\mathcal{N C}_{G_{32}} \cong \mathcal{N C} \mathcal{N}_{G(1,1,5)}$.

To verify whether or not a poset admits a symmetric chain decomposition by computer is usually a very time-consuming task, and we could only succeed to prove this property for noncrossing partition lattices associated with exceptional well-generated complex reflection groups of rank at most 3 . However, SAGE has an efficient algorithm to determine the maximum size of an antichain (or the width) of a given poset, and it is thus possible to check by computer if a poset is Sperner. In what follows we describe a strategy to reduce the question whether a poset is strongly Sperner to successively checking whether certain subposets are Sperner. In particular, this yields a feasible strategy to check whether a poset is strongly Sperner by computer.

Let $\mathcal{P}=(P, \leq)$ be a graded poset of length $n$ with rank vector $\mathbf{N}_{\mathcal{P}}=\left(N_{0}, N_{1}, \ldots, N_{n}\right)$. There is certainly some $r \in\{0,1, \ldots, n\}$ such that $N_{i} \leq N_{r}$ for all $i \in\{0,1, \ldots, n\}$. (This $r$ need not be unique.) Let $R=\{p \in P \mid \operatorname{rk}(p)=r\}$, and define $\mathcal{P}[1]=(P \backslash R, \leq)$. Moreover, define $\mathcal{P}[0]=\mathcal{P}$ and $\mathcal{P}[i]=(\ldots((\mathcal{P}[\underbrace{[1])[1]) \ldots)[1]}_{i}$. In other words, $\mathcal{P}[i]$ is the poset that is created from $\mathcal{P}$ by removing the $i$ largest ranks, and is consequently a graded poset in its own right. Clearly, $\mathcal{P}[s]$ is the empty poset for $s>n$. We have the following result. 
Proposition 15 A bounded poset $\mathcal{P}$ with $\ell(\mathcal{P})=n$ is strongly Sperner if and only if $\mathcal{P}[i]$ is Sperner for each $i \in\{0,1, \ldots, n\}$.

The weaker statement " $\mathcal{P}$ is $k$-Sperner if and only if $\mathcal{P}[i]$ is Sperner for each $i \in\{0,1, \ldots, k\}$ " is not true, as for instance the example in Figure 2 shows. If the poset in Figure 2(b) is denoted by $\mathcal{P}$, then the poset in Figure 2(c) is $\mathcal{P}[1]$. We observe that $\mathcal{P}$ is 1 -Sperner, but $\mathcal{P}[1]$ is not. Now we conclude the proof of Theorem 2

Proof of Theorem 2; First suppose that $W$ is irreducible. Theorem 3 implies together with Theorems 8 . 1.,13, and Proposition 14 that $\mathcal{N} \mathcal{C}_{W}$ is Peck, i.e. strongly Sperner, rank-symmetric, and rank-unimodal, whenever $W$ is isomorphic to $G(1,1, n)$ for $n \geq 1$, to $G(d, d, n)$ for $d, n \geq 2$, or to $G(d, 1, n)$ for $d \geq 2, n \geq 1$. The exceptional cases have been verified by computer using Proposition 15 .

If $W$ is reducible, then we have $W \cong W_{1} \times W_{2} \times \cdots \times W_{s}$ for some well-generated irreducible complex reflection groups $W_{i}$, and we can define a noncrossing partition lattice for $W$ by $\mathcal{N C}_{W} \cong \mathcal{N C}_{W_{1}} \times \mathcal{N C}_{W_{2}} \times$ $\cdots \times \mathcal{N C}_{W_{s}}$. The claim now follows from Theorem 5

\section{References}

[1] I. Anderson. Combinatorics of Finite Sets. Dover Publications, Mineola, NY, 2002.

[2] D. Armstrong. Generalized Noncrossing Partitions and Combinatorics of Coxeter Groups. Mem. Amer. Math. Soc., 202, 2009.

[3] C. A. Athanasiadis and V. Reiner. Noncrossing Partitions for the Group $D_{n}$. SIAM J. Discrete Math., 18:397-417, 2004.

[4] D. Bessis. The Dual Braid Monoid. Ann. Sci. École Norm. Sup., 36:647-683, 2003.

[5] D. Bessis. Finite Complex Reflection Arrangements are $K(\pi, 1)$. Ann. of. Math., 181:809-904, 2015.

[6] P. Biane. Some Properties of Crossings and Partitions. Discrete Math., 175:41-53, 1997.

[7] T. Brady. A Partial Order on the Symmetric Group and new $K(\pi, 1)$ 's for the Braid Groups. Adv. Math., 161:20-40, 2001.

[8] M. Broué, G. Malle, and R. Rouquier. Complex Reflection Groups, Braid Groups, Hecke Algebras. Journal für die reine und angewandte Mathematik, 500:127-190, 1998.

[9] E. R. Canfield. On A Problem of Rota. Adv. Math., 29:1-10, 1978.

[10] E. R. Canfield. A Sperner Property Preserved by Product. Linear and Multilinear Alg., 9:151-157, 1980.

[11] C. Chevalley. Invariants of Finite Groups Generated by Reflections. Amer. J. Math., 77:778-782, 1955.

[12] B. A. Davey and H. A. Priestley. Introduction to Lattices and Order. Cambridge University Press, Cambridge, 2002. 
[13] N. G. de Bruijn, C. A. van Ebbenhorst Tengbergen, and D. Kruyswijk. On the Set of Divisors of a Number. Nieuw Arch. Wiskd., 23:191-193, 1951.

[14] R. P. Dilworth and C. Greene. A Counterexample to the Generalization of Sperner's Theorem. J. Combin. Thery, 10:18-21, 1971.

[15] K. Engel. Sperner Theory. Cambridge University Press, Cambridge, 1997.

[16] P. Erdős. On a Lemma of Littlewood and Offord. Bull. Amer. Math. Soc., 51:898-902, 1945.

[17] J. R. Griggs. Symmetric Chain Orders, Sperner Theorems, and Loop Matchings. Ph.d. thesis, 1977.

[18] G. I. Lehrer and T. A. Springer. Reflection Subquotients of Unitary Reflection Groups. Canad. J. Math., 51:1175-1193, 1999.

[19] G. I. Lehrer and D. E. Taylor. Unitary Reflection Groups. Cambridge University Press, Cambridge, 2009.

[20] H. Mühle. EL-Shellability and Noncrossing Partitions Associated with Well-Generated Complex Reflection Groups. Europ. J. Combin., 43:249-278, 2015.

[21] H. Mühle. Symmetric Chain Decompositions and the Strong Sperner Property for Noncrossing Partition Lattices. arXiv:1509.06942, 2015.

[22] P. Orlik and L. Solomon. Unitary Reflection Groups and Cohomology. Invent. Math., 59:77-94, 1980.

[23] R. A. Proctor, M. E. Saks, and D. G. Sturtevant. Product Partial Orders with the Sperner Property. Discrete Math., 30:173-180, 1980.

[24] V. Reiner. Non-Crossing Partitions for Classical Reflection Groups. Discrete Math., 177:195-222, 1997.

[25] V. Reiner, V. Ripoll, and C. Stump. On Non-Conjugate Coxeter Elements in Well-Generated Reflection Groups. arXiv:1404.5522, 2014.

[26] G. C. Shephard and J. A. Todd. Finite Unitary Reflection Groups. Canad. J. Math., 6:274-304, 1954.

[27] R. Simion and D. Ullmann. On the Structure of the Lattice of Noncrossing Partitions. Discrete Math., 98:193-206, 1991.

[28] E. Sperner. Ein Satz über Untermengen einer endlichen Menge. Math. Zeitschrift, 27:544-548, 1928.

[29] T. A. Springer. Regular Elements of Finite Reflection Groups. Invent. Math., 25:159-198, 1974.

[30] R. P. Stanley. Weyl Groups, the Hard Lefschetz Theorem, and the Sperner Property. SIAM J. Alg. Disc. Meth., 1:168-184, 1980.

[31] R. P. Stanley. Quotients of Peck Posets. Order, 1:29-34, 1984. 